\title{
Competencias digitales de docentes en la educación superior
} universitaria: retos y perspectivas en el ámbito de la educación virtual

José Lisbinio Cruz Guimaraes josecruzguimaraes@gmail.com ORCID: 0000-0002-9497-0037 Magister en educación Con mención en docencia y gestión educativa Universidad Privada César Vallejo Perú

Brigitte Elizabeth Llantoy Aroca Brigittellantoyaroca3@gmail.com ORCID: 0000-0001-8473-3743

Mg. Docencia universitaria Universidad Privada César Vallejo

Manuel Jesús Guevara Martínez guevaramanuel2021@gmail.com ORCID: 0000-0001-6896-0399

$\mathrm{Mg}$. Docencia universitaria Universidad Privada César Vallejo

Andy Walter Rivera Reátegui Andyriverareategui@gmail.com ORCID: 0000-0002-8874-7154

$\mathrm{Mg}$. Docencia universitaria Universidad Privada César Vallejo

\section{RESUMEN}

Angélica María Minchola Vásquez mincholaangelica25@gmail.com ORCID: 0000-0001-8247-3650

Mg. Docencia universitaria Universidad Nacional de Piura

En el contexto actual afectado por la pandemia, la digitalización ha cumplido un rol fundamental en el ámbito académico, tanto para la continuidad de la actividad pedagógica y la innovación en las metodologías de enseñanza - aprendizaje, sin embargo, se han observado deficiencias propias de su aplicación por los actores del proceso que implican nuevas perspectivas y retos por cumplir. La investigación tuvo como objetivo examinar y especificar los retos y perspectivas para el dominio de las competencias digitales por parte de los docentes universitarios en el ámbito de educación virtual. La metodología aplicada fue a través de la revisión sistemática donde se recopiló literatura científica 
indexada a través de portales científicos confiables como Scopus, Science direct, Scielo, Redalyc, Ebsco y Researchgate, posteriormente la data fue procesada a través de criterios de inclusión, considerándose entre estos la relación directa con las variables, asimismo, que deben encontrarse entre un periodo de tiempo entre el 2016-2021 y en idioma inglés y español, donde finalmente se obtuvieron 15 artículos para el análisis. Se logró determinar la importancia de llevar a la práctica docente los aprendizajes adquiridos en materia de TICs, para así desarrollar de forma amplia las competencias digitales docentes, renovando y adaptando dichas habilidades a cada realidad, siendo ello el principal reto a superar, para alcanza los estándares educativos que se exigen en el siglo XXI.

Palabras clave: competencias digitales; docentes; educación superior; retos; TIC. 


\title{
Digital competences of teachers in university higher education: challenges and perspectives in the field of virtual education
}

\begin{abstract}
In the current context affected by the pandemic, digitalization has played a fundamental role in the academic sphere, both for the continuity of pedagogical activity and innovation in teaching methodologies - learning, However, there have been shortcomings in its implementation by the actors in the process that imply new perspectives and challenges to be met. The aim of the research was to examine and specify the challenges and perspectives for the mastery of digital skills by university teachers in the field of virtual education. The applied methodology was through the systematic review where indexed scientific literature was collected through reliable scientific portals such as Scopus, Science direct, Scielo, Redalyc, Ebsco and Researchgate, data was subsequently processed through inclusion criteria, which included the direct relationship with the variables, which must also be between a period of time between 2016 - 2021 and in English and Spanish, where 15 articles were finally obtained for analysis. It was possible to determine the importance of translating the learning acquired in the field of ICTs into teaching practice, in order to develop in a broad way the teaching digital competences, renewing and adapting these skills to every reality, This is the main challenge to overcome, in order to achieve the educational standards required in the 21 st century.
\end{abstract}

Keywords: digital skills; teachers; higher education; challenges; ICT.

Artículo recibido: 30 noviembre. 2021 Aceptado para publicación: 29 diciembre 2021 Correspondencia: josecruzguimaraes@gmail.com Conflictos de Interés: Ninguna que declarar 


\section{INTRODUCCIÓN}

En la actualidad, la exigencia de la sociedad y la influencia de las tecnologías digitales, han generado cambios importantes en la educación, especialmente sobre su rol fundamentado en el desarrollo del ser humano. Ramos (2017) mencionó que a través de la educación el individuo logra desarrollar habilidades, destrezas, determinación ética y moral que posteriormente será necesaria para adaptarse al entorno social donde se desenvuelve y además contribuir con la evolución de la misma. Por su parte, Martí, Montero y Sánchez (2018) mencionaron que la educación se ha convertido en la principal herramienta para el desarrollo social, asimismo, contribuye considerablemente con el perfeccionamiento cognitivo y personal del individuo. Debido a ello, esta se sitúa como uno de los elementos fundamentales para la civilización, lo cual amerita una consideración especial conforme a su aplicación práctica.

Es importante mencionar, que la educación al ser de vital importancia para la sociedad, requiere de un procedimiento idóneo que garantice la calidad de la misma, dado que para que exista una evolución notable, los individuos deben haber formado parte de un proceso de enseñanza - aprendizaje que mantenga todos los elementos necesarios para su desarrollo, sin embargo, este resultado no siempre es el esperado, debido a que el sistema educativo presenta múltiples variables que no se mantienen de manera homogénea.

Barba (2018) explicó que la calidad educativa depende de diversos factores, entre los que se encuentran los contenidos, metodologías, infraestructura, equidad y disposición de los docentes, estos elementos sincronizados adecuadamente permiten la consolidación de un proceso educativo armonizado y de calidad, asimismo, Quintana (2018) mencionó que para asegurar la calidad educacional, es importante también la implementación de políticas estatales que permitan brindar los espacios necesarios para un funcionamiento adecuado, además de mantener la gestión de los centros educativos. Por otro lado, Llopis y Tejerina (2016) señalaron que para poder medir el nivel de calidad educativa, se deben considerar factores demográficos que permitan conocer con mayor precisión la situación en la que los individuos se desenvuelven y como estos pueden afectar, considerando el nivel de ingresos económicos, la precariedad laboral, la salud y la vivienda.

Como se ha observado, la educación está sujeta a la interacción de diversos factores, los cuales intervienen en su proceso y en el resultado que esta pueda obtener, cabe mencionar 
que a medida que avanza el tiempo, surgen nuevos retos que demandan importantes cambios los cuales deben ser considerados por la actividad pedagógica.

Rodríguez et al (2016) explicaron que la llegada de la tecnología y el internet han mostrado un cambio importante en la vida de las personas, de la misma manera ha tenido un efecto considerable en el ámbito educativo, convirtiéndose en la actualidad en una herramienta de afianzamiento pedagógico, volviéndose cada vez más habitual entre estudiantes universitarios, de la misma manera, Argente et al (2017) señalaron que, los jóvenes se han adaptado rápidamente al enfoque tecnológico, a través del cual, han encontrado los medios para presentar mayor entusiasmo y actividad, además de convertirse en una herramienta de soporte metodológico para la enseñanza, complementándose con las redes sociales y facilitando el proceso de enseñanza aprendizaje, asimismo, Fuentes, Estrada y Delgado (2021) mencionaron que en la actualidad, la revolución industrial 4.0 exige la implementación de la digitalización en el ámbito educativo, de manera que a través de esta se logre impulsar el desarrollo de profesionales que dominen estas competencias, debido a ello, se han transformado los enfoques pedagógicos y académicos junto con experiencias didácticas a través de dispositivos y redes sociales.

Como se pudo observar, la tecnología se ha complementado con el ámbito educativo para brindar diversas perspectivas innovadoras, haciéndolas más didáctica e interactivas para los actores del proceso de enseñanza - aprendizaje. Es a través de esta que el docente puede captar la atención del estudiante de manera más facilitada y además podrá mejorar los resultados, por otro lado, el estudiante logrará desarrollar competencias digitales, las cuales son requeridas por el campo laboral actualmente, y así encontrarse apto para adaptarse a las nuevas exigencias.

Hoy en día, se han observado cambios radicales en el mundo, especialmente relacionados a la llegada el COVID - 19, el cual ha impulsado un direccionamiento obligatorio hacia la digitalización, dadas las restricciones que se impusieron por su expansión mundial.

Al respecto, Cervantes y Alvites (2021) afirmaron que la educación presentó cambios forzados, acelerados e importantes con la llegada del COVID - 19, el cual impuso un reto para el sistema, siendo afectados diversos sectores, comenzando por la imposición de la cuarentena que impidió la continuidad de las clases dentro de la modalidad presencial, debido a un aislamiento requerido. En función de ello, se observó mayor presencia de las 
Tecnologías de Información y Comunicación (TIC), a través de las cuales se ha implementado la enseñanza virtualizada, facilitada especialmente por los recursos y alternativas que estas herramientas ofrecen. Por otro lado, García (2021) señaló que el cierre de establecimientos educacionales y de aprendizaje han afectado a un $94 \%$ de la población estudiantil mundial, lo cual se ha visto más acentuado en países que presentan recursos limitados. Adicionalmente, Expósito y Marsollier (2020) mencionaron que existe cierto nivel de inequidad en esta modalidad, dado que a pesar de las ventajas que tiene, existe un grupo importante de estudiantes que no tienen acceso a las herramientas tecnológicas, lo cual interrumpe su proceso educativo.

Considerando lo expuesto, se puede mencionar que en la actualidad la tecnología y la digitalización cumplen un rol fundamental en la educación, convirtiéndose en el intermediario para que el proceso de enseñanza - aprendizaje pueda realizarse sin interrupciones pese a las restricciones ocasionadas por el COVID - 19; por otro lado, se han convertido en una herramienta que permite innovar con la metodología de enseñanza, debido a sus múltiples funciones, afianzando el aprendizaje y simplificando barreras de espacio y tiempo, que anteriormente se consideraban imposibles.

Debido a la importancia que ha tomado la digitalización, los docentes deben encontrarse capacitados para su manejo e inmediata implementación en las aulas, considerando que el dominio de las competencias tecnológicas y digitales es primordial sobretodo en la educación superior, para garantizar el desarrollo de habilidades que permitan su correcta aplicación por parte del estudiantado, en el mercado laboral, el cual es cada vez más exigente respecto al dominio de las mismas, dentro del perfil profesional.

Siendo así, se pudo observar durante el contexto de la pandemia, que la educación virtual fue transformándose en el principal medio para efectuar las prácticas educativas, por lo cual, se busca que los resultados obtenidos a través de esta nueva modalidad, sean tan eficientes como el sistema de educación presencial. Sin embargo, existen diversos retos para el sistema educativo, como la homogenización, el dominio de herramientas informáticas por parte de los docentes y la falta de equipamiento, entre otros.

Cabero, Barroso y Palacios (2020) explicaron que las competencias digitales son la base fundamental para la integración de las TIC en el proceso de enseñanza - aprendizaje, por lo cual, los docentes deben asumir el reto de capacitar a los estudiantes para su adaptación en un entorno cada vez más tecnificado, de manera que se requiere que se obtenga un 
manejo en las tic's mayor a lo registrado en la actualidad. Por su parte, Padilla et al (2019) mencionaron que entre los retos congruentes con las competencias digitales, se encuentra el dominio de las herramientas tecnológicas, manejo de dispositivos táctiles y material pedagógico digitalizado, el dominio de espacios virtuales, en reemplazo de los espacios físicos, de manera que, promover el desarrollo y dominio de las competencias digitales inicialmente por el docente garantizará que pueda darse inicio al proceso de enseñanza. Sin embargo, Martínez y Garcés (2020) señalaron que son pocos los docentes que obtienen la capacidad innovadora en competencias digitales, observándose que las que tienen mayor dominio son comunicación, colaboración y desarrollo de problemas, debido a ello, es necesaria la implementación de estrategias de fortalecimiento docente, en las asignaturas más específicas y/o especializadas.

En vista de estas deficiencias, se asume entre los principales retos y desafíos para los docentes de la educación superior universitaria, la alfabetización digital, el dominio de herramienta digitales especializadas, manejo de redes sociales, uso de instrumentos tecnológicos y manejo de plataformas para el desarrollo de clases virtuales. Todos estos elementos deben ser abordados dentro del contexto de la educación virtual de forma eficiente por parte de los docentes universitarios, para que los educandos logren desarrollar competencias virtuales, actualmente demandadas en el ámbito laboral.

Por otro lado, son diversas las perspectivas que traen consigo el dominio de las competencias digitales, especialmente sus enfoques en el campo educacional. Acevedo et al (2020) afirmaron que entre los principales enfoques, se encuentra la innovación, dado que permite lograr transformaciones importantes en las metodologías de enseñanza, optimizándolas, logrando ser más interactivas y eficientes para el proceso de educativo. En línea con ello, García, Ortiz y Chávez (2020) mencionaron que la perspectiva social también ha introducido cambios en las estrategias de enseñanza, de forma considerable, demandando a los docentes hacer uso ineludible de las TIC para mejorar su actividad académica, especialmente desde un entorno virtualizado. Asimismo, Cabero, Barroso y Palacios (2021) explicaron que otra de las perspectivas se basa en la evaluación de competencias, es decir, medir la capacidad que percibe el docente de sí mismo, logrando realizar comparativos en cuando al rendimiento de la actividad que realiza, motivándolo así a ser más competitivo. 
Ciertamente, son diversas las perspectivas en cuanto a las competencias digitales, pero en todas se observa una necesidad perenne por adaptarlas al contexto educativo, esta perspectiva se da debido a que en el contexto de la educación virtual forzada por la pandemia, el dominio de las herramientas digitales se ha convertido en una necesidad para los actores del proceso educacional, por lo cual, en la actualidad comprende un elemento clave para el desarrollo profesional y social. Es por ello, que el docente como elemento difusor de conocimientos, debe dominarlas para que de esta manera mejore su desempeño a través de metodologías innovadoras y logre así, transferir sus conocimientos a los estudiantes, alcanzando resultados transcendentales donde se vean reflejados los esfuerzos por elevar la calidad educativa, enfatizando la funcionabilidad de las competencias digitales en los docentes de educación superior universitaria.

\section{ESTRATEGIAS METODOLÓGICAS O MATERIALES Y MÉTODOS}

A fines de garantizar la veracidad y objetividad en el presente artículo, se ha tomado en consideración determinados criterios que han permitido compilar información de manera objetiva y enfocada a los fines de la misma, a través de lo cual se obtuvo una base de datos vasta, comprendida por literatura científica diversificada, la cual, posteriormente fue depurada de acuerdo a los criterios establecidos por el autor, priorizando la solidez del estudio.

En la elaboración de la investigación fue aplicado el método de revisión bibliográfica, a través del cual se realizó la búsqueda y recopilación de literatura científica y académica indexada, la cual está sujeta a criterios establecidos como ser de idioma inglés o español, la relación directa con las variables de investigación y encontrarse en el periodo de tiempo comprendido entre los años 2016 al 2021, para lo cual se realizaron estrategias de búsqueda empleando palabras clave como: Competencias digitales, tecnología, TIC, educación superior, metodologías, docentes, enseñanza, aprendizaje, siendo mencionadas de forma alternada, permitiendo obtener mayor amplitud en la búsqueda.

La exploración de información se dio por medio de buscadores científicos confiables como Scopus, Ebsco, Redalyc, Scielo, Science direct y Researchgate, posterior a la realización de la búsqueda se obtuvieron 40 artículos, los que fueron examinados de acuerdo a los criterios establecidos, obteniéndose como resultado 15 artículos seleccionados para el análisis de estudio. 
El proceso de análisis se realizó a través de la revisión particular de cada uno de los artículos seleccionados, los cuales fueron distribuidos en un archivo de Word por medio de tablas que permitieron organizarlos de manera más detallada y objetiva, donde se plantearon los núcleos temáticos de cada uno de los estudios analizados. 


\section{RESULTADOS Y DISCUSIÓN}

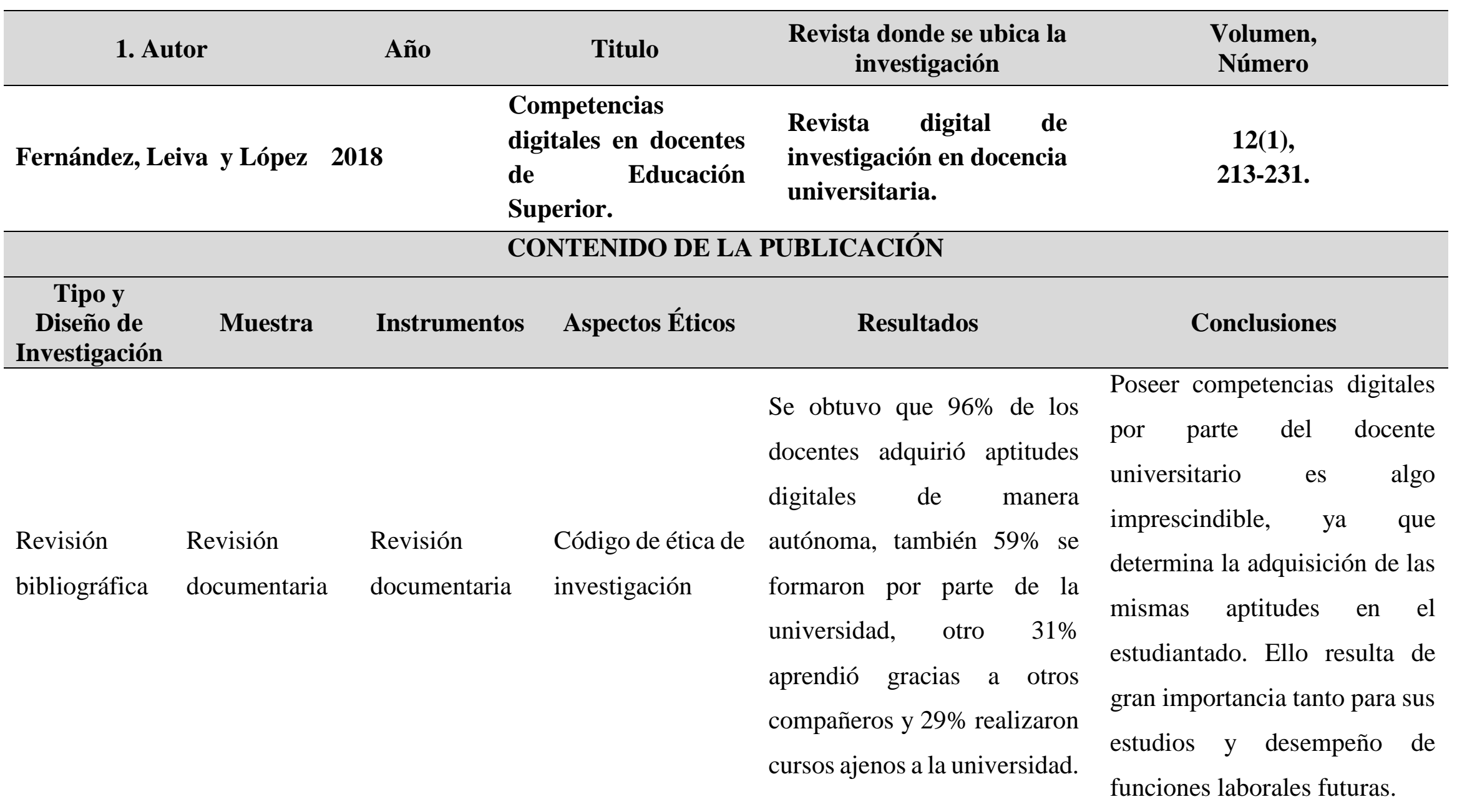




\begin{tabular}{|c|c|c|c|c|c|}
\hline \multicolumn{2}{|c|}{ 2. Autor } & Año & Titulo & $\begin{array}{c}\text { Revista donde se ubica la } \\
\text { investigación }\end{array}$ & $\begin{array}{l}\text { Volumen, } \\
\text { Número }\end{array}$ \\
\hline \multicolumn{2}{|l|}{ Pozos y Tejada } & $\begin{array}{l}\text { C } \\
\text { d } \\
\text { st } \\
\text { d } \\
\text { f }\end{array}$ & $\begin{array}{l}\text { Competencias digitales } \\
\text { docentes en educación } \\
\text { superior: niveles de } \\
\text { dominio y necesidades } \\
\text { formativas. }\end{array}$ & $\begin{array}{l}\text { Revista Digital de } \\
\text { Investigación en } \\
\text { Docencia Universitaria. }\end{array}$ & $\begin{array}{l}12(2) \\
59-87\end{array}$ \\
\hline \multicolumn{6}{|c|}{ CONTENIDO DE LA PUBLICACIÓN } \\
\hline $\begin{array}{c}\text { Tipo y } \\
\text { Diseño de } \\
\text { Investigación }\end{array}$ & Muestra & Instrumentos & Aspectos Éticos & Resultados & Conclusiones \\
\hline $\begin{array}{l}\text { Revisión } \\
\text { bibliográfica }\end{array}$ & $\begin{array}{l}\text { Revisión } \\
\text { documentaria }\end{array}$ & $\begin{array}{l}\text { Revisión } \\
\text { documentaria }\end{array}$ & $\begin{array}{l}\text { Código de ética de } \\
\text { investigación }\end{array}$ & $\begin{array}{l}\text { Se obtuvo que las necesidades } \\
\text { formativas con prioridad } \\
\text { "alta" están vinculadas con la } \\
\text { docencia, la investigación y la } \\
\text { gestión en ambientes } \\
\text { presenciales, mixtos y } \\
\text { virtuales. Estas altas } \\
\text { necesidades afectan los } \\
\text { niveles de profundización y } \\
\text { de generación de nuevos } \\
\text { conocimientos en los } \\
\text { estudiantes universitarios. }\end{array}$ & $\begin{array}{l}\text { Es fundamental atender la } \\
\text { formación pedagógica que } \\
\text { demanda el profesorado, } \\
\text { brindando acompañamiento } \\
\text { continuo para integrar } \\
\text { adecuadamente las TIC en la } \\
\text { tarea docente universitaria y así } \\
\text { garantizar que además de la } \\
\text { tecnología, cuenten con las } \\
\text { competencias digitales para } \\
\text { aprovecharlas en el proceso de } \\
\text { enseñanza y aprendizaje. }\end{array}$ \\
\hline
\end{tabular}




\begin{tabular}{|c|c|c|c|c|c|}
\hline \multicolumn{2}{|c|}{ 3. Autor } & \multicolumn{2}{|r|}{ Titulo } & $\begin{array}{c}\text { Revista donde se ubica la } \\
\text { investigación }\end{array}$ & $\begin{array}{l}\text { Volumen, } \\
\text { Número }\end{array}$ \\
\hline \multicolumn{2}{|c|}{ Padilla et al. } & $\begin{array}{l}\text { Co } \\
\text { Do } \\
\text { Su } \\
\text { Un } \\
\text { Alt }\end{array}$ & $\begin{array}{l}\text { npetencias Digitales } \\
\text { entes en Educación } \\
\text { erior: caso Centro } \\
\text { versitario de Los } \\
\text { ss. }\end{array}$ & $\begin{array}{l}\text { Revista de Educación } \\
\text { y Desarrollo. }\end{array}$ & $\begin{array}{c}1(51) \\
89-95 .\end{array}$ \\
\hline \multicolumn{6}{|c|}{ CONTENIDO DE LA PUBLICACIÓN } \\
\hline $\begin{array}{c}\text { Tipo y Diseño } \\
\text { de } \\
\text { Investigación }\end{array}$ & Muestra & Instrumentos & Aspectos Éticos & Resultados & Conclusiones \\
\hline $\begin{array}{l}\text { Revisión } \\
\text { bibliográfica }\end{array}$ & $\begin{array}{l}\text { Revisión } \\
\text { documentaria }\end{array}$ & $\begin{array}{l}\text { Revisión } \\
\text { documentaria }\end{array}$ & $\begin{array}{l}\text { Código de ética de } \\
\text { investigación }\end{array}$ & $\begin{array}{l}\text { Los resultados arrojaron que } \\
\text { el grado de competencia } \\
\text { digital de los profesores } \\
\text { universitarios es "bajo", sin } \\
\text { embargo, demuestran interés } \\
\text { por recibir formación para } \\
\text { mejorar sus habilidades } \\
\text { digitales. Asimismo, existe } \\
\text { un nivel "bajo" en cuanto al } \\
\text { "Uso y alfabetización } \\
\text { tecnológica". Además, el } \\
\text { tipo de género no afecta las } \\
\text { habilidades digitales. }\end{array}$ & $\begin{array}{l}\text { Se destacó la importancia de } \\
\text { contar con recursos tecnológicos } \\
\text { resaltando que ello no conlleva } \\
\text { de forma rápida a mejorar el } \\
\text { proceso de enseñanza } \\
\text { aprendizaje; siendo necesario } \\
\text { implementar cursos para que los } \\
\text { docentes utilicen las TIC's eficaz } \\
\text { y efectivamente, convirtiéndolas } \\
\text { en un elemento clave para lograr } \\
\text { reformas educativas de amplio } \\
\text { alcance, acorde con los retos } \\
\text { educativos actuales. }\end{array}$ \\
\hline
\end{tabular}




\begin{tabular}{|c|c|c|c|c|c|}
\hline \multicolumn{2}{|c|}{ 4. Autor } & \multicolumn{2}{|r|}{ Titulo } & $\begin{array}{c}\text { Revista donde se ubica la } \\
\text { investigación }\end{array}$ & $\begin{array}{l}\text { Volumen, } \\
\text { Número }\end{array}$ \\
\hline \multicolumn{2}{|c|}{ Acevedo et al. } & \multirow[t]{2}{*}{2020} & $\begin{array}{l}\text { Competencias } r \text { del } \\
\text { docente en educación } \\
\text { online en tiempo de } \\
\text { COVID-19: } \\
\text { Universidades Públicas } \\
\text { de Honduras. }\end{array}$ & $\begin{array}{l}\text { el } \\
\text { on } \\
\text { de }\end{array}$ & $\begin{array}{l}26(1) \\
1-18\end{array}$ \\
\hline \multicolumn{5}{|c|}{ CONTENIDO DE LA PUBLICACIÓN } & \\
\hline $\begin{array}{c}\text { Tipo y } \\
\text { Diseño de } \\
\text { Investigación } \\
\end{array}$ & Muestra & Instrumentos & Aspectos Éticos & Resultados & Conclusiones \\
\hline $\begin{array}{l}\text { Revisión } \\
\text { bibliográfica }\end{array}$ & $\begin{array}{l}\text { Revisión } \\
\text { documentaria }\end{array}$ & $\begin{array}{l}\text { Revisión } \\
\text { documentaria }\end{array}$ & $\begin{array}{l}\text { Código de ética } \\
\text { de investigación }\end{array}$ & $\begin{array}{l}\text { Las dificultades de los docentes } \\
\text { por el cambio de la modalidad } \\
\text { presencial a la virtual } \\
\text { representaron un } 50,3 \% \text { de } \\
\text { afectación, mientras que la } \\
\text { interacción en las plataformas } \\
\text { digitales con estudiantes, contó } \\
\text { con } 56,9 \% \text { de aceptación. Por } \\
\text { otra parte, 54,72\% utilizó el } \\
\text { WhatsApp como medio de } \\
\text { enlace y un } 28,33 \% \text { de los } \\
\text { docentes manejó la herramienta } \\
\text { zoom para cumplir con sus } \\
\text { clases. }\end{array}$ & $\begin{array}{l}\text { La "virtualización de la } \\
\text { planificación curricular" } \\
\text { representa un reto para la } \\
\text { educación superior y, según los } \\
\text { autores, tanto docentes como } \\
\text { estudiantes deben colaborar para } \\
\text { adaptarse a los cambios y ser } \\
\text { competitivos, siendo la principal } \\
\text { competencia digital a desarrollar: } \\
\text { una actitud positiva hacia las } \\
\text { TIC's, demostrando con ello, una } \\
\text { perspectiva innovadora, basada } \\
\text { en asimilar los cambios del } \\
\text { entorno. }\end{array}$ \\
\hline
\end{tabular}




\begin{tabular}{|c|c|c|c|c|c|}
\hline \multicolumn{2}{|c|}{ 5. Autor } & \multicolumn{2}{|r|}{ Titulo } & \multirow[t]{2}{*}{$\begin{array}{c}\text { Revista donde se ubica la } \\
\text { investigación }\end{array}$} & $\begin{array}{l}\text { Volumen, } \\
\text { Número }\end{array}$ \\
\hline $\begin{array}{r}\text { Cabero, I } \\
\text { Pala }\end{array}$ & $\begin{array}{l}\operatorname{arroso} y \\
\text { ios }\end{array}$ & $\begin{array}{l}\text { Es } \\
\text { co } \\
\text { do } \\
\text { la } \\
\text { co }\end{array}$ & $\begin{array}{l}\text { udio de la } \\
\text { npetencia digital } \\
\text { ente en Ciencias de } \\
\text { Salud. Su relación } \\
\text { algunas variables }\end{array}$ & & $\begin{array}{c}(22) \\
1\end{array}$ \\
\hline \multicolumn{6}{|c|}{ CONTENIDO DE LA PUBLICACIÓN } \\
\hline $\begin{array}{c}\text { Tipo y Diseño } \\
\text { de } \\
\text { Investigación }\end{array}$ & Muestra & Instrumentos & Aspectos Éticos & Resultados & Conclusiones \\
\hline $\begin{array}{l}\text { Revisión } \\
\text { bibliográfica }\end{array}$ & $\begin{array}{l}\text { Revisión } \\
\text { documentaria }\end{array}$ & $\begin{array}{l}\text { Revisión } \\
\text { documentaria }\end{array}$ & $\begin{array}{l}\text { Código de ética de } \\
\text { investigación }\end{array}$ & $\begin{array}{l}\text { Existe una diferencia notable } \\
\text { entre los docentes de } 50 \text { a } 59 \\
\text { años, con los docentes que se } \\
\text { encuentran entre } 30 \text { - } 39 \text { años, } \\
\text { siendo el segundo grupo el que } \\
\text { presenta mayor dominio de las } \\
\text { competencias digitales, mientras } \\
\text { que los docentes de } 40 \text { a } 49 \text { años } \\
\text { poseen dominio intermedio en } \\
\text { referencia a los grupos antes } \\
\text { mencionados. }\end{array}$ & $\begin{array}{l}\text { Se observa que los docentes que } \\
\text { se encuentran entre los } 30-49 \\
\text { años de edad son los que poseen } \\
\text { mayor dominio de competencias } \\
\text { digitales. Se observó que estas son } \\
\text { independientes del género. Por } \\
\text { otro lado, se observó que los } \\
\text { docentes que tienen experiencia } \\
\text { entre los } 4 \text { y } 14 \text { años son los que } \\
\text { muestran mayor dominio de las } \\
\text { competencias digitales. }\end{array}$ \\
\hline
\end{tabular}




\begin{tabular}{|c|c|c|c|c|c|}
\hline \multicolumn{2}{|c|}{ 6. Autor } & Año & Titulo & $\begin{array}{c}\text { Revista donde se ubica la } \\
\text { investigación }\end{array}$ & $\begin{array}{l}\text { Volumen, } \\
\text { Número }\end{array}$ \\
\hline \multicolumn{2}{|c|}{ Martínez y Garcés } & 2020 & $\begin{array}{l}\text { mpetencias digitales } \\
\text { entes y el reto de la } \\
\text { cación virtual derivado } \\
\text { la covid-19. }\end{array}$ & $\begin{array}{c}\text { Educación } \\
\text { y Humanismo }\end{array}$ & $\begin{array}{c}22(39) \\
1-16\end{array}$ \\
\hline \multicolumn{6}{|c|}{ CONTENIDO DE LA PUBLICACIÓN } \\
\hline $\begin{array}{c}\text { Tipo y Diseño de } \\
\text { Investigación }\end{array}$ & Muestra & Instrumentos & Aspectos Éticos & Resultados & Conclusiones \\
\hline $\begin{array}{l}\text { Revisión } \\
\text { bibliográfica }\end{array}$ & $\begin{array}{l}\text { Revisión } \\
\text { documentaria }\end{array}$ & $\begin{array}{l}\text { Revisión } \\
\text { documentaria }\end{array}$ & $\begin{array}{l}\text { Código de ética de } \\
\text { investigación }\end{array}$ & $\begin{array}{l}\text { De acuerdo a este estudio, se } \\
\text { obtuvo que las competencias } \\
\text { digitales bridan al } 78,85 \% \text { de } \\
\text { los docentes, herramientas para } \\
\text { clasificar contenidos digitales, } \\
\text { así como compartir } \\
\text { información en medios } \\
\text { virtuales: 50,00\%, editar } \\
\text { contenido digital } 40,38 \% \text {, } \\
\text { resguardar datos personales: } \\
40,38 \% \text { y desarrollar } \\
\text { competencias conceptuales: } \\
42,31 \% \text {. En ese sentido, existen } \\
\text { relaciones directas, entre tres } \\
\text { de las competencias. }\end{array}$ & $\begin{array}{l}\text { Según los autores, uno de los retos a } \\
\text { superar en el campo educativo } \\
\text { basado en las competencias digitales } \\
\text { es elevar los indicadores de } \\
\text { innovación e implementar } \\
\text { estrategias de capacitaciones } \\
\text { docentes en el uso de las Tics, } \\
\text { respaldando la calidad del servicio } \\
\text { educativo brindado por instituciones } \\
\text { públicas y privadas, dentro de un } \\
\text { contexto de crisis sanitaria } \\
\text { globalizadas, por lo que las } \\
\text { capacitaciones en competencias } \\
\text { digitales harán la diferencia en el } \\
\text { progreso educativo, económico y } \\
\text { social. }\end{array}$ \\
\hline
\end{tabular}




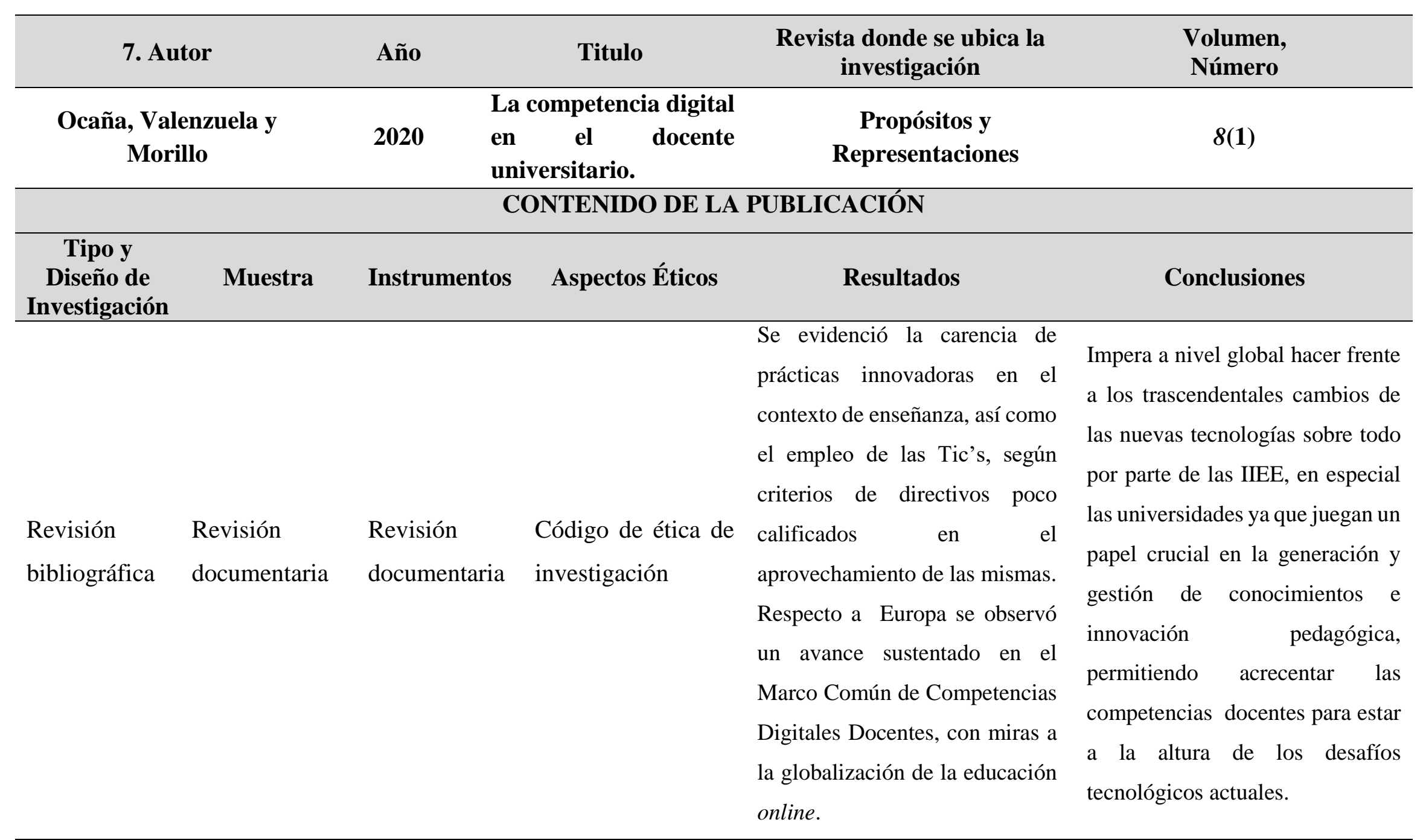




\begin{tabular}{|c|c|c|c|c|c|}
\hline \multicolumn{2}{|c|}{ 8. Autor } & Año & Titulo & $\begin{array}{c}\text { Revista donde se ubica la } \\
\text { investigación }\end{array}$ & $\begin{array}{l}\text { Volumen, } \\
\text { Número }\end{array}$ \\
\hline \multicolumn{2}{|c|}{$\begin{array}{c}\text { Perdomo, Martínez y } \\
\text { Barreto }\end{array}$} & \multirow[t]{2}{*}{2020} & $\begin{array}{l}\text { mpetencias } \\
\text { itales en docentes } \\
\text { iversitarios: una } \\
\text { isión sistemática de } \\
\text { iteratura }\end{array}$ & \multirow[b]{2}{*}{ PUBLICACIÓN } & \multirow[t]{2}{*}{$\begin{array}{c}9(2) \\
92-115\end{array}$} \\
\hline \multicolumn{3}{|c|}{ CONTENIDO DE LA PUBLICACIÓN } & & & \\
\hline $\begin{array}{c}\text { Tipo y } \\
\text { Diseño de } \\
\text { Investigación }\end{array}$ & Muestra & Instrumentos & Aspectos Éticos & Resultados & Conclusiones \\
\hline $\begin{array}{l}\text { Revisión } \\
\text { bibliográfica }\end{array}$ & $\begin{array}{l}\text { Revisión } \\
\text { documentaria }\end{array}$ & $\begin{array}{l}\text { Revisión } \\
\text { documentaria }\end{array}$ & $\begin{array}{l}\text { Código de ética de } \\
\text { investigación }\end{array}$ & $\begin{array}{l}\text { A través del análisis de } \\
\text { diversos estudios se observó } \\
\text { que las competencias digitales } \\
\text { en los docentes universitarios } \\
\text { han sido el centro de debates y } \\
\text { redefiniciones de prácticas y } \\
\text { roles, por lo que demanda el } \\
\text { establecer criterios para } \\
\text { determinar cuáles pueden ser } \\
\text { las propuestas, estrategias y } \\
\text { herramientas más propicias } \\
\text { para lograr objetivos } \\
\text { pedagógicos actuales. }\end{array}$ & $\begin{array}{l}\text { Basado en la recopilación de } \\
\text { diversos estudios, se concluyó que } \\
\text { las competencias digitales } \\
\text { corresponden a la capacidad de usar } \\
\text { las herramientas y recursos Tics } \\
\text { favoreciendo la búsqueda, análisis y } \\
\text { transformación de conocimientos de } \\
\text { manera objetiva, así como poder } \\
\text { trabajar en equipo, compartiendo } \\
\text { dichos conocimientos con ética y } \\
\text { responsabilidad, en la actividad } \\
\text { pedagógica, laboral y social, actual } \\
\text { y futura }\end{array}$ \\
\hline
\end{tabular}




\begin{tabular}{|c|c|c|c|c|c|}
\hline \multicolumn{2}{|c|}{ 9. Autor } & Año & Titulo & $\begin{array}{c}\text { Revista donde se ubica la } \\
\text { investigación }\end{array}$ & $\begin{array}{l}\text { Volumen, } \\
\text { Número }\end{array}$ \\
\hline Colás, Conc & e y Reyes & \multicolumn{2}{|c|}{$\begin{array}{l}\text { Sustainability and } \\
\text { Digital Teaching } \\
\text { Competence in Higher } \\
\text { Education. }\end{array}$} & Sustainability & $\begin{array}{l}13(22) \\
12354\end{array}$ \\
\hline \multicolumn{6}{|c|}{ CONTENIDO DE LA PUBLICACIÓN } \\
\hline $\begin{array}{c}\text { Tipo y } \\
\text { Diseño de } \\
\text { Investigación } \\
\end{array}$ & Muestra & Instrumentos & Aspectos Éticos & Resultados & Conclusiones \\
\hline $\begin{array}{l}\text { Revisión } \\
\text { bibliográfica }\end{array}$ & $\begin{array}{l}\text { Revisión } \\
\text { documentaria }\end{array}$ & $\begin{array}{l}\text { Revisión } \\
\text { documentaria }\end{array}$ & $\begin{array}{l}\text { Código de ética de } \\
\text { investigación }\end{array}$ & $\begin{array}{l}\text { Las competencias digitales } \\
\text { pedagógicas más relevantes } \\
\text { según este estudio, son, la } \\
\text { creación de contenidos digitales } \\
\text { con } 28,4 \% \text {, pedagogía digital } \\
\text { con } 20,9 \% \text { basado en las } \\
\text { habilidades en el uso de } \\
\text { herramientas Tics para innovar y } \\
\text { colaborar en el aprendizaje } \\
\text { autodirigido y finalmente, la } \\
\text { capacidad docente para } \\
\text { empoderar a los estudiantes y } \\
\text { fomentar la inclusión }(13,4 \%) \text {. }\end{array}$ & $\begin{array}{l}\text { Se determinó que las } \\
\text { competencias digitales están } \\
\text { vinculadas a criterios de } \\
\text { sostenibilidad internacional, } \\
\text { basado en una perspectiva de } \\
\text { evolución pedagógica a través del } \\
\text { empleo de recursos tecnológicos, } \\
\text { identificando la naturaleza de las } \\
\text { competencias digitales útiles en la } \\
\text { práctica universitaria, según el } \\
\text { modelo DigComp Edu, } \\
\text { promoviendo la calidad educativa. }\end{array}$ \\
\hline
\end{tabular}




\begin{tabular}{|c|c|c|c|c|c|}
\hline \multicolumn{2}{|c|}{ 10. Autor } & \multicolumn{2}{|r|}{ Titulo } & \multirow[t]{2}{*}{$\begin{array}{c}\text { Revista donde se ubica la } \\
\text { investigación }\end{array}$} & \multirow[t]{2}{*}{$\begin{array}{l}\text { Volumen, } \\
\text { Número }\end{array}$} \\
\hline \multicolumn{2}{|c|}{ Fernández et al . } & 2021 & $\begin{array}{l}\text { Digital Teaching } \\
\text { Competence in Higher } \\
\text { Education: A } \\
\text { Systematic Review. }\end{array}$ & & \\
\hline \multicolumn{6}{|c|}{ CONTENIDO DE LA PUBLICACIÓN } \\
\hline $\begin{array}{c}\text { Tipo y } \\
\text { Diseño de } \\
\text { Investigación } \\
\end{array}$ & Muestra & Instrumentos & Aspectos Éticos & Resultados & Conclusiones \\
\hline $\begin{array}{l}\text { Revisión } \\
\text { bibliográfica }\end{array}$ & $\begin{array}{l}\text { Revisión } \\
\text { documentaria }\end{array}$ & $\begin{array}{l}\text { Revisión } \\
\text { documentaria }\end{array}$ & $\begin{array}{l}\text { Código de ética de } \\
\text { investigación }\end{array}$ & $\begin{array}{l}\text { Se evidenció } \\
\text { obstáculos que impiden el } \\
\text { desarrollo de competencias } \\
\text { digitales docentes: falta de } \\
\text { formación del profesorado } \\
(30 \%) \text {, bajo perfil (20\%), la falta } \\
\text { de experiencia (15\%) o falta de } \\
\text { recursos (10\%). Otras barreras } \\
\text { son: falta de tiempo (10\%) y } \\
\text { falta de apoyo de instituciones } \\
(5 \%) \text {, problemas económicos } \\
(5 \%) \text { y problemas de acceso a } \\
\text { Internet (5\%). }\end{array}$ & $\begin{array}{l}\text { Las competencias digitales } \\
\text { docente está cobrando mayor } \\
\text { relevancia en los últimos años, } \\
\text { favoreciendo el desarrollo de } \\
\text { producción científica de cara al } \\
\text { siglo XXI, contando así con } \\
\text { diversos análisis exhaustivos para } \\
\text { desarrollar entornos de } \\
\text { aprendizaje consolidados por las } \\
\text { TICs. Representandocon ello, una } \\
\text { mejora en los procesos de } \\
\text { enseñanza y aprendizaje a nivel } \\
\text { global. }\end{array}$ \\
\hline
\end{tabular}




\begin{tabular}{|c|c|c|c|c|c|}
\hline \multicolumn{2}{|c|}{ 11. Autor } & Año & Titulo & $\begin{array}{c}\text { Revista donde se ubica la } \\
\text { investigación }\end{array}$ & $\begin{array}{l}\text { Volumen, } \\
\text { Número }\end{array}$ \\
\hline \multirow{2}{*}{\multicolumn{2}{|c|}{ García, Ortiz y Chávez }} & 2021 & $\begin{array}{l}\text { Relevancia y dominio } \\
\text { de las competencias } \\
\text { digitales del docente } \\
\text { en la educación } \\
\text { superior. }\end{array}$ & $\begin{array}{c}\text { Revista Cubana de } \\
\text { Educación Superior. }\end{array}$ & $40(3)$. \\
\hline & & \multicolumn{4}{|c|}{ CONTENIDO DE LA PUBLICACIÓN } \\
\hline $\begin{array}{c}\text { Tipo y } \\
\text { Diseño de } \\
\text { Investigación }\end{array}$ & Muestra & Instrumentos & Aspectos Éticos & Resultados & Conclusiones \\
\hline $\begin{array}{l}\text { Revisión } \\
\text { bibliográfica }\end{array}$ & $\begin{array}{l}\text { Revisión } \\
\text { documentaria }\end{array}$ & $\begin{array}{l}\text { Revisión } \\
\text { documentaria }\end{array}$ & $\begin{array}{l}\text { Código de ética de } \\
\text { investigación }\end{array}$ & $\begin{array}{l}\text { Se observó que los sistemas } \\
\text { digitales brindan un enfoque } \\
\text { novedoso en el contexto } \\
\text { educativo, personal y social, } \\
\text { evidenciando así la influencia } \\
\text { de dichas tendencias digitales } \\
\text { en la evolución en los modelos } \\
\text { de enseñanza aprendizaje, } \\
\text { demandando capacitaciones en } \\
\text { competencias digitales } \\
\text { docentes, necesarias para } \\
\text { afrontar los cambios } \\
\text { pedagógicos actuales. }\end{array}$ & $\begin{array}{l}\text { Las perspectivas de estrategias de } \\
\text { enseñanza - aprendizaje deben ser } \\
\text { abordadas desde la capacitación en } \\
\text { competencias digitales docentes, } \\
\text { como única vía para afrontar las } \\
\text { disyuntivas educativas, partiendo } \\
\text { por la alfabetización digital a nivel } \\
\text { universitario, previendo la extinción } \\
\text { de pedagogías y profesiones que no } \\
\text { se alinean con el paradigma } \\
\text { tecnológico actual, ideado bajo las } \\
\text { TIC y las diversas plataformas de } \\
\text { interactividad. }\end{array}$ \\
\hline
\end{tabular}




\begin{tabular}{|c|c|c|c|c|}
\hline 12. Autor & Año & Titulo & $\begin{array}{c}\text { Revista donde se ubica la } \\
\text { investigación }\end{array}$ & $\begin{array}{l}\text { Volumen, } \\
\text { Número }\end{array}$ \\
\hline Martín et al. & 2021 & $\begin{array}{lr}\begin{array}{l}\text { Metodología } \\
\text { trabajo }\end{array} & \begin{array}{r}\text { de } \\
\text { con }\end{array} \\
\text { universidades } \\
\text { públicas de Perú } \\
\text { durante la pandemia. }\end{array}$ & Ciencias de la educación & $\begin{array}{l}11(7) \\
351 .\end{array}$ \\
\hline
\end{tabular}

\begin{tabular}{|c|c|c|c|c|c|}
\hline \multicolumn{6}{|c|}{ CONTENIDO DE LA PUBLICACIÓN } \\
\hline $\begin{array}{c}\text { Tipo y } \\
\text { Diseño de } \\
\text { Investigación }\end{array}$ & Muestra & Instrumentos & Aspectos Éticos & Resultados & Conclusiones \\
\hline $\begin{array}{l}\text { Revisión } \\
\text { bibliográfica }\end{array}$ & $\begin{array}{l}\text { Revisión } \\
\text { documentaria }\end{array}$ & $\begin{array}{l}\text { Revisión } \\
\text { documentaria }\end{array}$ & $\begin{array}{l}\text { Código de ética de } \\
\text { investigación }\end{array}$ & $\begin{array}{l}\text { Se evidenció que la falta de } \\
\text { conectividad por parte de los } \\
\text { estudiantes fue la mayor } \\
\text { afectación en la adaptación del } \\
\text { contexto de educación } \\
\text { presencial al modelo virtual, } \\
\text { mientras que, en la dimensión de } \\
\text { competencias digitales docentes, } \\
\text { se observó falta de } \\
\text { conocimientos pedagógicos y } \\
\text { digitales entre los profesores } \\
\text { universitarios. }\end{array}$ & $\begin{array}{l}\text { Los cambios causados por la crisis } \\
\text { sanitaria y la creciente } \\
\text { virtualidad, afectaron a las } \\
\text { universidades nacionales } \\
\text { convirtiendo en reto el continuar } \\
\text { su labor educativa, empleando } \\
\text { por tanto, todos los recursos } \\
\text { disponibles y crucialmente las } \\
\text { TICs, permitiendo así definir } \\
\text { planes de acción para garantizar } \\
\text { la adaptación a los cambios } \\
\text { globalizados. }\end{array}$ \\
\hline
\end{tabular}




\begin{tabular}{|c|c|c|c|c|c|}
\hline \multicolumn{2}{|c|}{ 13. Autor } & Año & Titulo & $\begin{array}{c}\text { Revista donde se ubica la } \\
\text { investigación }\end{array}$ & $\begin{array}{l}\text { Volumen, } \\
\text { Número }\end{array}$ \\
\hline Pérez, Priet & y Miguel & $\begin{array}{l}\mathrm{A} \\
\mathrm{C} \\
\mathrm{S} \\
\mathrm{P} \\
\mathrm{E} \\
\mathrm{S} \\
1\end{array}$ & $\begin{array}{l}\text { Analysis of Digital } \\
\text { Competence for } \\
\text { Spanish Teachers at } \\
\text { Pre-University } \\
\text { Educational Key } \\
\text { Stages during COVID- } \\
\text { 19. }\end{array}$ & $\begin{array}{c}\text { International Journal of } \\
\text { Environmental Research } \\
\text { and Public Health. 18(15), } \\
8093 .\end{array}$ & $\begin{array}{l}18(15) \\
8093 .\end{array}$ \\
\hline \multicolumn{6}{|c|}{ CONTENIDO DE LA PUBLICACIÓN } \\
\hline $\begin{array}{c}\text { Tipo y Diseño } \\
\text { de } \\
\text { Investigación }\end{array}$ & Muestra & Instrumentos & Aspectos Éticos & Resultados & Conclusiones \\
\hline $\begin{array}{l}\text { Revisión } \\
\text { bibliográfica }\end{array}$ & $\begin{array}{l}\text { Revisión } \\
\text { documentaria }\end{array}$ & $\begin{array}{l}\text { Revisión } \\
\text { documentaria }\end{array}$ & $\begin{array}{l}\text { Código de ética de } \\
\text { investigación }\end{array}$ & $\begin{array}{l}\text { Se demostró que los profesores } \\
\text { con menor edad y poca } \\
\text { trayectoria profesional (12 años } \\
\text { de labor), hacen un mayor uso } \\
\text { de las herramientas TICs en un } \\
43.69 \% \text { en promedio. Contrario } \\
\text { a ello, los docentes con mayor } \\
\text { experiencia laboral (18 años en } \\
\text { promedio) no hacen uso de las } \\
\text { TICs en un 48,56\%. En total } \\
70 \% \text { del grupo demostró poseer } \\
\text { conocimiento medio-alto. }\end{array}$ & $\begin{array}{l}\text { Es preocupante que los docentes } \\
\text { reflejen un alto nivel de uso de las } \\
\text { redes sociales, más sin embargo, no } \\
\text { poseen competencias digitales que } \\
\text { le permitan emplearlos en su labor } \\
\text { docente, siendo actualmente un reto } \\
\text { a vencer en este contexto, ya que son } \\
\text { instrumentos comunicativos } \\
\text { ampliamente utilizados por los } \\
\text { estudiantes en sus actividades } \\
\text { sociales, por lo que su uso en la } \\
\text { educación puede ser una motivación } \\
\text { novedosa y simple. }\end{array}$ \\
\hline
\end{tabular}




\begin{tabular}{|c|c|c|c|c|c|}
\hline \multicolumn{2}{|c|}{ 14. Autor } & Año & Titulo & $\begin{array}{l}\text { Revista donde se ubica } \\
\text { la investigación }\end{array}$ & $\begin{array}{l}\text { Volumen, } \\
\text { Número }\end{array}$ \\
\hline \multicolumn{2}{|c|}{ Cruzado, et al. } & 2021 & $\begin{array}{l}\text { álisis de competencias } \\
\text { sitales de docentes de } \\
\text { provincia de } \\
\text { siones: el reto de la } \\
\text { eva alfabetización. }\end{array}$ & $\begin{array}{c}\text { Virtualidad, Educación y } \\
\text { Ciencia }\end{array}$ & $\begin{array}{c}12(23) \\
8-32\end{array}$ \\
\hline \multicolumn{6}{|c|}{ CONTENIDO DE LA PUBLICACIÓN } \\
\hline $\begin{array}{c}\text { Tipo y } \\
\text { Diseño de } \\
\text { Investigación }\end{array}$ & Muestra & Instrumentos & Aspectos Éticos & Resultados & Conclusiones \\
\hline $\begin{array}{l}\text { Revisión } \\
\text { bibliográfica }\end{array}$ & $\begin{array}{l}\text { Revisión } \\
\text { documentaria }\end{array}$ & $\begin{array}{l}\text { Revisión } \\
\text { documentaria }\end{array}$ & $\begin{array}{l}\text { Código de ética de } \\
\text { investigación }\end{array}$ & $\begin{array}{l}\text { Se demostró que un } 27.6 \% \\
\text { del profesorado respondió } \\
\text { que conoce y utiliza "algo" } \\
\text { de las competencias digitales, } \\
\text { otro } 24,8 \% \text { señalaron "La } \\
\text { conozco y la utilizo poco". } \\
\text { Finalmente, un } 20,3 \% \\
\text { manifestó conocer las } \\
\text { competencias digitales pero } \\
\text { no lo utilizarlas. Adicional, se } \\
\text { obtuvo mejor percepción de } \\
\text { las competencias digitales por } \\
\text { parte del género masculino. }\end{array}$ & $\begin{array}{l}\text { Como perspectiva, se concluyó que } \\
\text { una adecuada preparación y } \\
\text { alfabetización digital en los } \\
\text { docentes permitirá transferir estas } \\
\text { habilidades y conocimientos a los } \\
\text { estudiantes, lo que fomentará a su } \\
\text { vez un fuerte sentido de "ciudadanía } \\
\text { digital" en las próximas } \\
\text { generaciones, de allí la importancia } \\
\text { de asumir el reto de capacitar para } \\
\text { perfeccionar las competencias } \\
\text { digitales de los docentes, para } \\
\text { afrontar satisfactoriamente } \\
\text { contextos globalizados. }\end{array}$ \\
\hline
\end{tabular}




\begin{tabular}{|c|c|c|c|c|c|}
\hline \multicolumn{2}{|c|}{ 15. Autor } & Año & Titulo & $\begin{array}{c}\text { Revista donde se ubica } \\
\text { la investigación }\end{array}$ & $\begin{array}{l}\text { Volumen, } \\
\text { Número }\end{array}$ \\
\hline \multicolumn{2}{|c|}{ González et al. } & $\begin{array}{l}\text { E } \\
\text { p } \\
\text { c: } \\
\text { sc } \\
\text { cc } \\
\text { el } \\
\#\end{array}$ & $\begin{array}{l}\text { Evaluación } r \text { de } \\
\text { programas online de } \\
\text { capacitación docente } \\
\text { sobre innovación y } \\
\text { competencias digitales } \\
\text { en la Covid-19: } \\
\text { \#webinarsUNIA }\end{array}$ & $\begin{array}{l}\text { Revista Iberoamericana de } \\
\text { Educación a Distancia }\end{array}$ & $\begin{array}{c}25(1) \\
121-140 .\end{array}$ \\
\hline \multicolumn{6}{|c|}{ CONTENIDO DE LA PUBLICACIÓN } \\
\hline $\begin{array}{c}\text { Tipo y } \\
\text { Diseño de } \\
\text { Investigación } \\
\end{array}$ & Muestra & Instrumentos & Aspectos Éticos & Resultados & Conclusiones \\
\hline $\begin{array}{l}\text { Revisión } \\
\text { bibliográfica }\end{array}$ & $\begin{array}{l}\text { Revisión } \\
\text { documentaria }\end{array}$ & $\begin{array}{l}\text { Revisión } \\
\text { documentaria }\end{array}$ & $\begin{array}{l}\text { Código de ética de } \\
\text { investigación }\end{array}$ & $\begin{array}{l}\text { Este estudio demostró que la } \\
\text { formación docente a través de } \\
\text { los webinars permite fortalecer } \\
\text { las competencias digitales, } \\
\text { basado en: Mejorar la } \\
\text { adaptación ante los nuevos } \\
\text { escenarios virtuales de } \\
\text { enseñanza-aprendizaje. Elevar } \\
\text { la calidad de las clases. } \\
\text { Ampliar los conocimientos } \\
\text { sobre la docencia virtual, y } \\
\text { Emplear aplicativos } \\
\text { tecnológicos educativos. }\end{array}$ & $\begin{array}{l}\text { Los programas de capacitación } \\
\text { virtual a través de los webinars } \\
\text { representan una oportunidad de } \\
\text { aprendizaje flexible y práctico que } \\
\text { benefician ampliamente a los } \\
\text { docentes por su accesibilidad y bajo } \\
\text { costo. Dicha apertura brinda la } \\
\text { posibilidad de obtener credenciales } \\
\text { de participación, apuntando al } \\
\text { mejoramiento de las competencias } \\
\text { docentes digitales que permiten al } \\
\text { docente renovarse y aplicar } \\
\text { estrategias educativas actuales. }\end{array}$ \\
\hline
\end{tabular}




\section{DISCUSIÓN}

Una vez culminada la revisión exhaustiva de los artículos científicos seleccionados, inherentes a las Competencias digitales de docentes en la educación superior universitaria, publicados en idioma español y varios en inglés, los mismos fueron referenciados de forma cronológica tomando como punto focal, los retos y perspectivas en el ámbito de la educación virtual, relacionando a la vez entornos culturales y contextos de crisis sanitarias. De esta manera pudo desarrollarse esta investigación de tipo descriptiva, siguiendo el modelo de las tablas de discusión donde se mencionaron uno a uno los 15 artículos, y así se logró valorar la trascendencia e impacto de las competencias digitales docentes desde el enfoque de distintos. En ese sentido, se logró identificar en gran medida las ventajas de desarrollar estas habilidades por los profesores, complementando las estrategias pedagógicas universitarias, siendo también importante en ese aspecto, la intervención oportuna de las entidades competentes para lograr que estas capacitaciones se logren de manera exitosa, tomando en cuenta adaptar y flexibilizar los modelos educativos virtuales, de acuerdo a la realidad y contexto de cada región y país, orientándolos a cumplir los objetivos académicos propuestos.

De acuerdo con Fernández, Leiva y López (2018), las competencias digitales docentes conllevan a una mejor planificación didáctica, pedagógica y también metodológica, diseñada para innovar la acción de enseñanza- aprendizaje en las aulas, sirviendo como guía en este nuevo contexto virtual, favoreciendo con ello, un autoaprendizaje en los jóvenes. También Pozos (2018) y Padilla et al. (2019), consideraron en su análisis, que la unificación de la competencia digital con el entorno universitario, comprende un gran compromiso por parte del docente con su propia mejora continua personal y profesional. Asimismo, Acevedo et al. 2020, asintieron que las competencias del docente en la educación online, deben ser lideradas y promocionadas por las universidades como instituciones educativas creadoras y transformadoras de contendidos pedagógicos, es allí donde está su principal reto, servir como puente entre las generaciones de docentes analógicos del siglo XIX y la generación de docentes digitalizados que demanda el siglo XX1.

Continuando con Cabero, Barroso y Palacios (2020), se destacó el impacto de las competencias digitales, de forma acrecentada, durante la crisis sanitaria, redimensionando la importancia de hacer de cada profesional un individuo capaz de 
comprender y desenvolverse en formatos virtuales, partiendo por los docentes ya que así pueden transferir las competencias digitales a los ciudadanos del mañana, continuando con los profesionales de la salud, diseñando planes de formación específicos en cada contexto, siguiendo metodologías digitalizadas. Asimismo, Martínez y Garcés (2020), señalaron que se requiere un desarrollo de competencias digitales docentes para crear un modelo educativo innovador en el campo pedagógico universitario, el cual aporte estrategias que motiven a los docentes y estudiantes durante el confinamiento por la COVID-19. Por su parte, Ocaña, Valenzuela y Morillo (2020), se plantearon la siguiente pregunta ¿por qué se hacen necesarias las competencias digitales del docente universitario para el actual contexto? Ante ello, se plantearon la respuesta de que dichas habilidades van a favorecer, en gran escala, a las nuevas generaciones denominadas "nativos digitales", siendo los canales digitales los preferidas para logar sus aprendizajes. De igual manera, el estudio realizado por Perdomo, Martínez y Barreto (2020), planteó entre sus argumentos, cinco áreas a desarrollar dentro de las competencias digitales: información y alfabetización informacional, creación de contenido digital, comunicación y colaboración, resolución de problemas y seguridad. Basado en ello, debe priorizarse la formación docente buscando la optimización e innovación de las competencias digitales en los profesores universitarios. Conjuntamente, se resaltó a Colás, Conde y Reyes (2021), quienes manifestaron "technology has been shown to make university systems more sustainable, as they provide inclusion and attention to diversity" [la tecnología ha demostrado que los sistemas universitarios son más sostenibles, ya que proporcionan inclusión y atención a la diversidad] fortaleciendo en ese aspecto, los principios universales que establecen el derecho a una educación de calidad, para todos por igual.

Por su parte, Fernández et al. (2021), plantearon otro enfoque en su investigación, argumentando que "university teaching staff are not sufficiently qualified in digital competences and are therefore not able to adapt their teaching methods to the demands of the current situation" [el personal docente universitario no logra adaptar sus métodos de enseñanza de acuerdo a las exigencias pedagógicas actuales, debido a que no está suficientemente cualificado en competencias digitales], y ello representa un reto, basado en redefinir y reajustar los métodos pedagógicos, lo cual no todos los docentes logran superar. Asimismo, García, Ortiz y Chávez (2021), señalaron que los cambios pedagógicos alienados con el desarrollo de competencias digitales, demanda de parte del 
docente un compromiso mayor, centrado en la motivación y determinación por lograr el dominio de las competencias digitales para emplearlas exitosamente en la dinámica educativa a nivel superior. También, Martín et al. (2021), señalaron que las estrategias de formación docente deben ser respaldadas por las propias universidades, sin embargo, resaltó la importancia de que los profesores brinden su tiempo para recibir la formación en competencias digitales, según sus necesidades personales y profesionales. En ese sentido, se evidenció una sensibilización e interés por desarrollar las competencias digitales, por parte de los profesores que formaron parte de este estudio.

Finalmente, Cruzado, et al. 2021, al cierre de su investigación resaltaron que la “alfabetización digital” permitirá que los docentes puedan derivar a sus estudiantes, más y mejores herramientas digitales, infiriendo que, siempre que estén los maestros y profesores, más capacitados en dichas competencias didácticas, podrán desarrollar un elevando sentido de "ciudadanía digital en los estudiantes". González et al. (2022) plantearon una perspectiva práctica para afrontar las limitaciones económicas que puedan impedir el desarrollo de las competencias digitales docentes, creando programas de talleres por videoconferencia o webinars, iniciados como una herramienta comunicativa, sin embargo, es actualmente, una estrategia institucionalizada, orientada en atender las necesidades formativas de docentes relacionados con el dominio de las Tics, retando y motivando así a la participación del profesorado, en ambientes virtuales dinámicos. Mientras que el estudio de Pérez, Prieto y Miguel (2021), propuso entre sus aportaciones, realizar más estudios sobre competencias digitales docentes, puntualizando con mayor precisión; ¿cuáles son las políticas de formación del profesorado de las universidades españolas?, siendo este, un enfoque interesante a investigar ya que no abordado en los estudios analizados.

\section{CONCLUSIÓN O CONSIDERACIONES FINALES}

Las fuentes literarias analizadas, evidenciaron, como a través del tiempo, distintas propuestas científicas y/o tecnológicas permitieron transformar las crisis en oportunidades, y los tiempos actuales no son la excepción. Por ello, al finalizar este estudio se determinó que, ante los entornos de incertidumbre como los que se viven actualmente, es congruente plantearnos dos alternativas: preparación y acción, ya que de nada sirve accionar sino se tiene una preparación adecuada y alineada con la realidad y contexto presente, y de igual manera, la preparación sin el accionar, ciertamente, no 
conduce a nada. Partiendo de ello, las capacitaciones en competencias digitales, llevadas a la práctica de manera continua, permitirán a los docentes universitarios afrontar los cambios vertiginosos que caracterizan a las nuevas tecnologías, en sus distintas dimensiones.

Al respecto Levano (2019) determinó que "la universidad necesita urgentemente académicos, organizativos, humanistas y científicos transformacionales, o no será capaz de enfrentar las nuevas perspectivas de la era digital", por tanto, se debe tener presente que, aun cuando la realidad plantee más preguntas que respuestas, los docentes deben siempre enfocarse en transformar su realidad, tomando en cuenta sus conocimientos y experiencia previas, unido al trabajo colaborativo, lo cual le permitirá saber con claridad qué, cómo y cuáles actividades deberá llevar a cabo para cumplir su rol académico, permitiéndose así alcanzar los objetivos de desarrollo personal y profesional en beneficio de elevar la calidad educativa de los estudiantes y aportar al progreso económico y social colectivo.

En ese orden, este estudio pudo lograr el objetivo planteado al inicio del mismo: examinar y especificar los retos y perspectivas para el dominio de las competencias digitales por parte de los docentes universitarios en el ámbito de educación virtual, obteniendo en función de ello diversas perspectivas que permitieron fortalecer habilidades cognitivas gracias a los aportes realizados por los diversos autores. De esta manera, se podrán identificar las fortalezas y debilidades en la labor docente actual y por tanto, establecer las bases para diseñar propuestas de formación orientadas a mejorar y perfeccionar la práctica docente en el aula, basado en el desarrollo profesional pedagógico en competencias digitales. Al respeto Lohr et al, (2021), señalaron que es trascendental por parte de los docentes, realizar una actualización de los conocimientos en uso de las TICs, y transformar así la práctica docente, teniendo total acceso al conocimiento de metodologías virtuales que garanticen el desarrollo de competencias digitales, en ellos.

Por lo anterior, y como nota de cierre, se alude a Quevedo (2020), quien destacó en su investigación, la importancia de emplear estrategias pedagógicas no solo tecnológicas sino también holísticas, por tanto este estudio plantea a las futuras investigaciones, empalmar las investigaciones sobre competencias digitales con el compendio de competencias holísticas, para lograr impartir e impulsar nuevas estrategias didácticas, garantizando de esa manera el contar con sistemas educativos inclusivos y de calidad que 
logren a través de una educación diversificada, motivar el desarrollo de personal y profesional tanto de estudiantes, como de profesores, dominando disciplinas y habilidades como la intuición, la emoción, la imaginación, y la capacidad creativa, sin dejar de lado, las competencias tecnológicas, para lograr una mejor resolución de conflictos, actuales y a futuro.

\section{LISTA DE REFERENCIAS}

Acevedo, A., Argüello, A., Pineda, B. y Urcios, P. (2020). Competencias del docente en educación online en tiempo de COVID-19: Universidades Públicas de Honduras. Revista de Ciencias Sociales. $26 \quad(1), \quad 1 \quad$ - 18. https://www.redalyc.org/articulo.oa? id=28064146014

Argente, E., Vivancos, E., Alemany, J. y García, A. (2017). Educando en privacidad en el uso de las redes sociales. Education in the knowledge society. 18 (2), 107 - 126. https://doi.org/10.14201/eks2017182107126

Barba, J. (2018). La calidad de la educación. RMIE. 23 (78), 963 - 979. http://www.scielo.org.mx/pdf/rmie/v23n78/1405-6666-rmie-23-78-963.pdf

Cabero, J., Barroso, J. y Palacios, A. (2020). Estudio de la competencia digital docente en Ciencias de la Salud. Su relación con algunas variables. Educación Médica, 22 (1), 94 -98. https://doi.org/10.1016/j.edumed.2020.11.014

Cervantes, C. y Alvites, C. (2021). WhatsApp como recurso educativo y tecnológico en la educación. Hamut'ay, 8 (2), 69-78, http://dx.doi.org/10.21503/hamu.v8i2.2294

Colás, P., Conde, J., y Reyes, S. (2021). Sustainability and Digital Teaching Competence in Higher Education. Sustainability, 13(22), 12354. https://www.mdpi.com/2071-1050/13/22/12354

Cruzado, C., Bonetti, S., Compaña, M., y Campión, R. (2021). Análisis de competencias digitales de docentes de la provincia de Misiones: el reto de la nueva alfabetización. Virtualidad, Educación y Ciencia, 12(23), 8-32. https://dialnet.unirioja.es/servlet/articulo? codigo $=8080293$

Fernández, E., Leiva, J. y López, E. (2018). Competencias digitales en docentes de Educación Superior. Revista digital de investigación en docencia universitaria, 12(1), 213-231.

http://www.scielo.org.pe/scielo.php?script=sci_arttext\&pid=S222325162018000100013 
Fernández, J., Román, P., Montenegro, M., López, E., Fernández, J. (2021). Digital Teaching Competence in Higher Education: A Systematic Review. Educ. Sci. 2021,11, 689. https://doi.org/10.3390/educsci11110689

Fuentes, D., Estrada, O. y Delgado, N. (2021). Las redes sociales digitales: una valoración socioeducativa. Revisión sistemática. Revista fuentes. 23 (1), 41 - 52. https://doi.org/10.12795/revistafuentes.2021.v23.i1.11947

García, K., Ortiz, T., y Chávez, M. (2021). Relevancia y dominio de las competencias digitales del docente en la educación superior. Revista Cubana de Educación Superior, 40(3).

http://scielo.sld.cu/scielo.php?script=sci_arttext\&pid=S0257-

43142021000300020

García, L. (2021). COVID-19 y educación a distancia digital: preconfinamiento, confinamiento y posconfinamiento. Revista iberoamericana de educación a distancia. 1 (24), 9 - 25.

https://www.redalyc.org/jatsRepo/3314/331464460001/331464460001.pdf

Levano, L., Sanchez, S., Guillén, P., Tello, S., Herrera, N., y Collantes, Z. (2019). Digital Competences and Education. Journal of Educational Psychology-Propositos y Representaciones, 7(2), 579-588.

https://files.eric.ed.gov/fulltext/EJ1220548.pdf

Llopis, R. y Tejerina, B. (2016). Crisis, educación y precariedad - afluencia. El rol de la educación en las condiciones de vida de la población española. Política y $\begin{array}{llllll}\text { sociedad. } & 53 & (2), & 413 & - & 442 .\end{array}$ https://doi.org/10.5209/rev_POSO.2016.v53.n2.49370

Lohr, A., Stadler, M., Schultz, F., Chernikova, O., Sailer, M., Fischer, F., y Sailer, M. (2021). On powerpointers, clickerers, and digital pros: Investigating the initiation of digital learning activities by teachers in higher education. Computers in Human Behavior, 119, 106715.

https://www.sciencedirect.com/science/article/pii/S0747563221000376

Martí, Y., Montero, B. y Sánchez, K. (2018). La función social de la educación: referentes teóricos actuales. Conrado. $14 \quad$ (63), $\quad 1 \quad-\quad 8$. http://scielo.sld.cu/scielo.php?script=sci_arttext\&pid=S199086442018000300259 
Martín, A, Lavandera, S., Mora, B., Sánchez, C., y Pérez L. (2021). Metodología de trabajo con universidades públicas de Perú durante la pandemia: continuidad de la enseñanza y el aprendizaje virtual / en línea. Ciencias de la educación, 11 (7), 351. https://www.mdpi.com/2227-7102/11/7/351

Martínez, J. y Garcés, J. (2020). Competencias digitales docentes y el reto de la educación virtual derivado de la covid-19. Educación y Humanismo, 22(39), 1-16. http://revistas.unisimon.edu.co/index.php/educacion/article/view/4114

Ocaña, Y., Valenzuela, L., y Morillo (2020). La competencia digital en el docente universitario. Propósitos y Representaciones, 8(1). http://www.scielo.org.pe/scielo.php?pid=S230779992020000200016\&script=sci_abstract\&tlng=en

Padilla, J., Ayala, G., Mora, O. y Ruezga, A. (2019). Competencias Digitales Docentes en Educación Superior: caso Centro Universitario de Los Altos. Revista de educación y desarrollo. 1 (51), 89 - 95.

Perdomo, B., Martínez, O. y Barreto, I.. (2020). Competencias digitales en docentes universitarios: una revisión sistemática de la literatura. EDMETIC, 9(2), 92-115. https://www.uco.es/ucopress/ojs/index.php/edmetic/article/view/12796

Pérez, E., Prieto, J., Miguel, V. (2021). Analysis of Digital Competence for Spanish Teachers at Pre-University Educational Key Stages during COVID-19. International Journal of Environmental Research and Public Health, 18(15), 8093. https:// doi.org/10.3390/ijerph18158093

Pozos, K, y Tejada, J. (2018). Competencias digitales docentes en educación superior: niveles de dominio y necesidades formativas. Revista Digital de Investigación en $\begin{array}{llll}\text { Docencia } & \text { Universitaria, } & \text { 59-87. }\end{array}$ http://dx.doi.org/10.19083/ridu.2018.712.

http://www.scielo.org.pe/pdf/ridu/v12n2/a04v12n2.pdf

Quevedo-Lezama, C. (2020). La Educación Holística: Una Oportunidad para Transformar la Realidad Educativa en el Siglo XXI. EDU REVIEW. International Education and Learning Review/Revista Internacional de Educación y Aprendizaje, 8(3), 165-179.

https://journals.gkacademics.com/revEDU/article/view/2522 
Quintana, Y. (2018). Calidad educativa y gestión escolar: una relación dinámica. Educ. $\begin{array}{llllll}\text { Educ. } & 21 & (2), & 259 & - & 281 .\end{array}$ http://www.scielo.org.co/pdf/eded/v21n2/0123-1294-eded-21-02-00259.pdf

Ramos, M. (2018). El rol de la educación y su contribución en la construcción de una sociedad mejor. Revista ensayos pedagógicos. 13 (1), 1 - 10. 10.15359/rep.13-1.1

Rodríguez, C., Valerio, G., Cárdenas, C., y Herrara, D. (2016). Percepción y realidad del uso de WhatsApp en estudiantes universitarios de ciencias de la salud. FEM. 19 (3), $119-124$. 